\title{
Philosophiques
}

\section{" To bring Dedekind's research into its proper relation to general metaphysical inquiry " : Royce et Russell, critiques de Bradley}

\section{Sébastien Gandon}

Volume 36, numéro 1, printemps 2009

L’idéalisme britannique

URI : https://id.erudit.org/iderudit/038011ar

DOI : https://doi.org/10.7202/038011ar

\section{Aller au sommaire du numéro}

\section{Éditeur(s)}

Société de philosophie du Québec

ISSN

0316-2923 (imprimé)

1492-1391 (numérique)

Découvrir la revue

Citer cet article

Gandon, S. (2009). « To bring Dedekind's research into its proper relation to general metaphysical inquiry " : Royce et Russell, critiques de Bradley. Philosophiques, 36(1), 83-108. https://doi.org/10.7202/038011ar

\section{Résumé de l'article}

Dans l'Appendice au livre I de The World and the Individual (1898), le philosophe américain Josiah Royce développe, en se fondant sur Was sind und was sollen die Zahlen? de Dedekind, une critique détaillée du livre de Bradley Appearance and Reality. Se concentrant sur le fameux § 66, Royce maintient que la théorie de Dedekind peut être vue comme l'accomplissement du mouvement de pensée inauguré par Fichte et Hegel : le « Soi idéal » est infini et l'arithmétique est la théorie de sa structure formelle. Ce texte curieux et négligé est intéressant pour au moins deux raisons. En premier lieu, la critique que Royce adresse à Bradley ressemble à celle de Russell : les deux auteurs fondent leur rejet du scepticisme bradleyien sur les mathématiques de Cantor et de Dedekind ; cependant, au lieu de rompre avec l'idéalisme, Royce plaide pour un retour à l'idéalisme rationnel de Fichte et de Hegel. Je présenterai les raisons qu'il a de procéder de la sorte et montrerai, contre l'opinion de Russell, qu'elles ne sont pas sans force. La seconde raison de lire Royce est que ce texte offre une interprétation originale, purement métaphysique, de la pensée de Dedekind. Je soutiendrai, à la fin de l'article, que cette analyse n'est pas sans pertinence, et que le philosophe allemand $\mathrm{H}$. Lotze, un collègue de Dedekind à Göttingen, pourrait avoir été le canal par lequel des thèmes issus de l'idéalisme hégélien et fichtéen sont parvenus jusqu’à Dedekind. 


\title{
«To bring Dedekind's research into its proper relation to general metaphysical inquiry $\gg$ : Royce et Russell, critiques de Bradley
}

\author{
SÉBASTIEN GANDON \\ Université Blaise Pascale, Clermont-Ferrand
}

\begin{abstract}
RÉSUMÉ. - Dans l'Appendice au livre I de The World and the Individual (1898), le philosophe américain Josiah Royce développe, en se fondant sur Was sind und was sollen die Zahlen? de Dedekind, une critique détaillée du livre de Bradley Appearance and Reality. Se concentrant sur le fameux $₫ 66$, Royce maintient que la théorie de Dedekind peut être vue comme l'accomplissement du mouvement de pensée inauguré par Fichte et $\mathrm{Hegel}$ : le «Soi idéal » est infini et l'arithmétique est la théorie de sa structure formelle. Ce texte curieux et négligé est intéressant pour au moins deux raisons. En premier lieu, la critique que Royce adresse à Bradley ressemble à celle de Russell: les deux auteurs fondent leur rejet du scepticisme bradleyien sur les mathématiques de Cantor et de Dedekind; cependant, au lieu de rompre avec l'idéalisme, Royce plaide pour un retour à l'idéalisme rationnel de Fichte et de Hegel. Je présenterai les raisons qu'il a de procéder de la sorte et montrerai, contre l'opinion de Russell, qu'elles ne sont pas sans force. La seconde raison de lire Royce est que ce texte offre une interprétation originale, purement métaphysique, de la pensée de Dedekind. Je soutiendrai, à la fin de l'article, que cette analyse n'est pas sans pertinence, et que le philosophe allemand $\mathrm{H}$. Lotze, un collègue de Dedekind à Göttingen, pourrait avoir été le canal par lequel des thèmes issus de l'idéalisme hégélien et fichtéen sont parvenus jusqu'à Dedekind.
\end{abstract}

ABSTRACT. - In the Appendice of The World and the Individual (1898), the American philosopher Josiah Royce grounds a lengthy and detailed critique of Bradley's book Appearance and Reality on Dedekind's work Was sind und was sollen die Zahlen? Focusing on the famous $\ 66$, Royce's main claim is that Dedekind's theory can be seen as a development of a line of thought first outlined by Fichte and Hegel: the so-called "ideal Self" is infinite, and arithmetic is the theory of its bare structural form. This curious but neglected work seems interesting for two reasons. Firstly, Royce's criticism of Bradley strongly reminds us of Russell's position: it is from the mathematical works of Dedekind and Cantor that the two writers put forward to Bradley's scepticism; however, instead of departing from idealism, Royce advocates a return to rational idealism. I will present his reasons to do that, and will try to show, against Russell's opinion, that they are not without strength. The second motive for going through Royce's work is that it offers a very original, purely metaphysical, interpretation of Dedekind's thought. I will claim, at the end of this paper, that Royce's analysis has some points for it, and that the German philosopher H. Lotze, a colleague of Dedekind in Göttingen, could have been the channel by which topics from Hegelian and Fichtean idealism have been conveyed to the German mathematician.

"To bring Dedekind's research into its proper relation to general metaphysical enquiry» est une phrase que le philosophe américain Josiah Royce écrit à 
la page 527 du supplément à The World and the Individual (1898), intitulé The One, the Many, and the Infinite. Le supplément, qui fait lui-même près de cent pages, se présente comme une critique du livre de Bradley Appearance and Reality (1893), fondée sur une lecture précise de Was sind und was sollen die Zablen?

On sait, mais j'y reviendrai, que le nerf de la critique adressée par Russell à Bradley consiste à distinguer deux sortes de régression à l'infini : une, très fréquente, mais inoffensive dès que l'on accepte, avec Cantor et Dedekind, le concept d'infini actuel; l'autre, plus problématique, mais plus rare, qui met en cause la notion même de "The meaning ». Selon Russell, Bradley, qui considère la notion d'infini comme contradictoire, confondrait constamment les deux sortes de régression et cette confusion serait à l'origine de la plupart des pseudo-apories de Appearance and Reality. Ce sont donc les mathématiques de Cantor et de Dedekind qui fournissent à Russell l'instrument de sa rupture avec Bradley et l'idéalisme de ses jeunes années.

L'intérêt du texte de Royce est de montrer que la même source aurait pu conduire le philosophe anglais vers d'autres rivages. Royce s'appuie en effet sur Dedekind pour affirmer la possibilité d'un nouvel idéalisme rationaliste. "Mettre les recherches de Dedekind dans leur contexte métaphysique propre» ne signifie pas, pour lui, substituer à l'idéalisme de Bradley un platonisme réaliste, mais lui substituer un nouvel idéalisme, peut-être d'ailleurs plus proche de Fichte et de Hegel que ne l'est celui de Bradley. Comme on le verra, Royce fonde son interprétation sur le concept non psychologique du Soi tel qu'il apparaît dans le $\$ 66$ de Was sind und was sollen die Zablen?

La source des deux critiques de Bradley, antagonistes dans leur esprit, est donc la même: les théories profondes et novatrices de Cantor et de Dedekind. Comment expliquer qu'un même socle mathématique conduise à des interprétations philosophiques aussi divergentes? L'une des deux lectures estelle intenable? Ou bien ne peut-on pas, à partir de Royce, remettre en question l'idée russellienne selon laquelle le réalisme issu de Moore serait la seule philosophie possible pour les mathématiques de l'époque? Nous décrirons, dans une première section, les critiques convergentes que Russell et Royce adressent à Bradley, avant de nous intéresser, dans la seconde partie, à l'usage métaphysique que Royce fait de Dedekind.

Mais en lisant The One, the Many, and the Infinite, une autre question émerge naturellement. L'interprétation métaphysique que Royce propose, à rebours de toute une tradition de lecture, purement internaliste, de Dedekind (qui remonte en France au grand livre de P. Dugac ${ }^{1}$ ), n'a-t-elle pas finalement de quoi se défendre? Ne peut-on pas déceler, dans plusieurs passages de Dedekind, comme un écho à certaines problématiques et à certaines prises de position idéalistes? Nous ne ferons ici qu'effleurer ce point, en nous interro- 
geant, dans un troisième temps, sur la pertinence historique de l'interprétation proposée par Royce. Des remarques plus générales sur le rapport entre mathématiques et métaphysique chez Bradley, Russell et Royce, concluront le propos.

\section{Russell et Royce, critiques de Bradley}

Russell et Royce critiquent tous les deux chez Bradley ce que l'on peut décrire comme une forme de scepticisme ou d'irrationalisme. Bradley n'est pas un

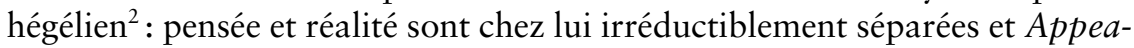
rance and Reality se déclinent comme une succession de vaines tentatives pour les saisir dans leur unité. Aucune conciliation finale, aucun savoir absolu ne vient «harmoniser» deux pôles qui demeurent, tout au long de l'ouvrage, constamment opposés.

Le début du chapitre XV de Bradley, 1893, intitulé Thought and Reality, dont Royce reprend l'analyse au début de son opuscule, illustre particulièrement bien la position bradleyenne. Si nous considérons quoi que ce soit de réel, nous trouvons en lui deux aspects (Bradley, 1893, p. 143):

Il y a un «ce que» [what] et un "que»[that $]$, une existence et un contenu, et les deux sont inséparables. Qu'une chose quelconque soit mais ne soit cependant rien de particulier, ou qu'une qualité ne qualifie et ne donne un caractère à aucune chose que ce soit, cela est évidemment impossible. Si nous cherchons à atteindre le «que » en lui-même, nous ne l'atteindrons pas, car, ou bien nous le qualifions, ou bien nous échouons complètement. Si nous cherchons à atteindre le «ce que » par lui-même, nous découvrons immédiatement qu'il n'est pas tout. Il pointe vers quelque chose au-delà de lui et ne peut pas exister par luimême et comme un simple adjectif. Aucun de ses aspects, si on les isole, ne peut être considéré comme réel, ou alors aucun d'eux, dans ce cas, ne demeure encore lui-même. Ils sont simplement distinguables, et non pas séparables.

La réalité, telle qu'elle est saisie dans la perception sensible, ne distingue pas l'être d'une chose de ses qualités, l'existence de son contenu. L'expérience sensible s'offre comme une totalité, comme une unité, au sein de laquelle différents aspects sont bien distinguables, mais non pas séparables, de la donnée du tout de l'expérience. Or la pensée est ce qui divise ce qui, dans la réalité (c'est-à-dire, pour Bradley, dans le sentir), forme

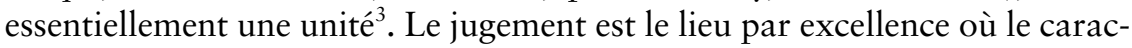
tère diabolisant de la pensée se manifeste. Comme Bradley s'en explique, la forme judicatoire distingue le sujet, qui est un "what», du prédicat, qui est un «that» (Bradley, 1893, p. 144-145):

2. Sur ce point, voir Allard, 2005.

3. Voir Bradley, 1893, p. 143-144: «La pensée semble essentiellement consister en leur séparation. Car la pensée, jusqu'à un certain point au moins, est idéale. Sans une idée, il n’y a aucune pensée, et une idée implique la séparation du contenu et de l'existence. C'est un «ce que » qui, dans la mesure où il est une simple idée, n'est pas, et qui, s'il était, ne pourrait pas, dans cette mesure même, être appelé idéal. Car l'idéalité consiste dans la disjonction de la qualité et de l'être.» 
Dans le jugement, une idée est prédiquée de la réalité. Or, en premier lieu, ce qui est prédiqué n'est pas une image mentale. [...] Le prédicat est un simple "ce que ", une simple caractéristique du contenu, qui est utilisé pour qualifier un peu mieux le «que» du sujet. [...] Le prédicat est un contenu qui a été détaché [had been made loose from] de sa propre existence immédiate, et qui est utilisé en tant que séparé de cette première unité [is used in divorce from that first unity].

Et en second lieu, lorsque nous nous tournons vers le sujet du jugement, nous découvrons clairement l'autre aspect, en d'autres mots, le "que». De même que dans «ce cheval est un mammifère » le prédicat n'est pas un fait, de même, et de façon encore plus certaine, le sujet est une existence réelle. [...] Personne ne cherche jamais à asserter quelque chose, si ce n'est de la réalité - Personne ne cherche jamais à faire quelque chose si ce n'est de qualifier un «que» par un «ce que». [...] Le jugement est essentiellement la réunion de deux faces, un «ce que» et un «que», provisoirement désunis. Mais c'est l'éloignement de ces aspects qui constitue l'idéalité de la pensée.

Les deux dernières phrases de la citation sont particulièrement claires: le jugement réunit deux aspects complémentaires de la réalité qui ont été artificiellement séparés l'un de l'autre. La forme même de l'unité judicatoire est donc, en son fondement même, différente de la forme d'unité qui s'attache à l'être saisi dans la perception sensible. Certes, le jugement réunit "deux faces ", mais cette réunion n'est que le prolongement du mouvement inaugural de scission entre le "what» et le «that» en lequel consiste l'idéalité (c.-à-d. l'essence même) du penser.

La leçon que Bradley tire est la suivante: aucun jugement, en tant qu'il est un jugement et sépare donc ce qui dans la réalité n’est pas séparé, n’est absolument vrai - la réalité ne peut être atteinte par la pensée. Il faudrait, bien entendu, pour être complet, analyser ici les conceptions bradleyiennes de la vérité (pour quelques éléments sur ce point, voir le texte de N. Griffin dans ce volume). Mais pour nous, l'important est de souligner le fait que le "scepticisme" de Bradley repose entièrement sur une certaine description objective de la pensée, comme activité de séparation entre un "that» et un "what», entre un sujet et un prédicat ${ }^{4}$. Nous pouvons résumer les diverses oppositions élaborées par Bradley dans un tableau à trois lignes:

4. En ce sens, ce que nous avons appelé «scepticisme » bradleyen diffère complètement du scepticisme ou du phénoménalisme humien. Bradley se montre ainsi très soucieux de distinguer les prédicats des images mentales. La confusion est pour lui à l'origine de l'idée selon laquelle le "what» a une existence réelle, et que la forme du jugement reflète donc finalement une forme (psychologique) de réalité. On assiste là à un curieux renversement: s'il faut critiquer le psychologisme de Hume, ce n'est pas, selon Bradley, pour échapper au scepticisme, mais au contraire, parce que, en identifiant les «what» ou "meaning» à des états mentaux existants, il pourrait nous en faire sortir! 


\begin{tabular}{|c|c|c|}
\hline \multicolumn{3}{|c|}{ Unité de l'expérience perceptive } \\
\hline Idéalité du penser & $\begin{array}{c}\text { That, Existence, } \\
\text { Reality, Being }\end{array}$ & $\begin{array}{c}\text { What, Content, } \\
\text { Ideality, Thought }\end{array}$ \\
\hline Jugement & \multicolumn{2}{|c|}{$\begin{array}{c}\text { Réunion du that et du what, qui } \\
\text { demeurent différents }\end{array}$} \\
\hline
\end{tabular}

La première ligne caractérise la réalité telle qu'elle est en son unité, c'est-à-dire telle qu'elle s'offre concrètement dans l'appréhension sensible. Les seconde et troisième lignes correspondent à la sphère de la pensée, qui sépare des aspects complémentaires de la réalité sensible avant de les réunir de façon superficielle dans le jugement.

En partant des mêmes travaux, ceux de Cantor et de Dedekind, Russell et Royce vont contester la validité d'un tel schéma. Les deux penseurs vont opposer à l'analyse de Bradley l'existence, en mathématique, de formes de pensée qui n'entrent pas dans le cadre mis en place par le philosophe. Cette parenté est reconnue par Bradley lui-même, qui, dans un article paru dans Mind en 1910, intitulé On Appearance, Error and Contradiction, associe les deux critiques: elles proviennent, dit-il, toutes deux de l'étude des mathématiques, mathématiques dont il se déclare «ignorant, non par choix mais par incapacité radicale" (Bradley, 1914], p. 277). Bradley, dans sa réponse, cherche à retrouver dans les concepts et procédures exposés par Royce et Russell ses propres catégories, celles du "what» et du "that» notamment, et donc à reconduire ses apories ${ }^{5}$.

Avant de détailler l'analyse de Royce, penchons-nous brièvement sur la critique, célèbre, de Russell. L'attaque se concentre sur un argument, toujours ressassé par Bradley: celui de l'impossibilité de la régression à l'infini. Ainsi, pour affirmer le caractère contradictoire du concept de relation, Bradley explique qu' "une relation reliant deux termes doit être reliée à chacun d'entre eux » et qu'en conséquence, pour pouvoir penser qu'une relation lie quelque terme que ce soit, il faut admettre une infinité de relations, ce qui est, selon Bradley, absurde. Russell reconnaît la validité de la première partie de l'argument: si une relation $R$ relie deux termes $a$ et $b$, alors deux nouvelles relations $R_{a}$ et $R_{b}$ doivent lier $R$ et $a$ et $R$ et $b$, ce qui amorce une régression à l'infini'. Mais il refuse le second moment: le concept d'infini n'a rien d'absurde ni de contradictoire. Qu'une proposition en implique une infinité d'autres ne constitue pas une objection à l'encontre de cette proposition, à partir du moment où ladite proposition peut être saisie indépendamment de

5. Russell et Royce ont correspondu l'un avec l'autre; Russell reprend d'ailleurs l'exemple de la carte de Royce (voir infra.) dans Russell, 1921.

6. Voir Russell, 1903, $\mathbb{9 9 9}$; voir également Allard, 2005. 
l'ensemble des propositions qu'elle implique. Russell distingue en effet une régression à l'infini dans l'implication, bénigne, d'une régression à l'infini dans le "meaning», plus problématique, parce que touchant à la structure même du terme .

C'est donc, selon Russell, parce que Bradley considère le concept d'infini comme contradictoire qu'il rejetterait la réalité des relations. La théorie que Cantor expose dans (Cantor, 1897) fournit les moyens de définir l'infini de façon positive. Une classe est infinie lorsqu'elle est, explique Cantor, similaire à une de ses parties propres, c.-à-d. lorsque, à chaque élément de la classe, correspond un et un seul élément de la partie considérée (voir infra. pour une analyse plus détaillée du concept). Dans cette définition, le concept d'infini n'est plus, comme dans la tradition aristotélicienne, lié à celui de négation et d'inachèvement, mais à une propriété, facilement exprimable, de certains ensembles. Bradley crierait au loup trop souvent ${ }^{8}$ : l'apparition de l'infini dans un raisonnement n'indique pas nécessairement la présence d'une contradiction.

Reste cependant un problème pour Russell: que l'infini puisse faire l'objet d'une définition ne signifie pas nécessairement qu'il y ait des classes infinies. Comment Russell peut-il être certain que la notion, telle qu'elle est définie par Cantor, désigne bien quelque chose? Cette question est celle que soulève Dedekind dans la lettre à Keferstein ${ }^{9}$. Russell, dans les Principles, reste curieusement évasif sur ce point, peut-être précisément parce que la fréquentation de Bradley l'a convaincu que le moindre bout de raisonnement dissimulait des régressions infinies. Dans le $\mathbb{} 339$ (Russell, 1903), le philosophe affirme ainsi: "Le fait qu'il y ait des classes infinies est tellement évident que l'on peut difficilement le nier. » Suivent deux références. La première au Parménide de Platon, qui suggère la preuve d'existence suivante: "Admettons qu'il y ait un nombre 1 . Alors 1 est, ou a de l'être, et en conséquence, il y a un être. Mais 1 et l'Être sont deux: ainsi, il y a un nombre 2; etc. ». La seconde au $\$ 13$ des Paradoxes de l'infini, où, dit Russell, Bolzano prouve, en corrélant chaque concept à l'idée d'un concept, que le nombre des concepts est infini (Voir infra. pour une analyse de l'argument de Bolzano et de sa reprise par Dedekind).

On le sait, les paradoxes auront raison de ces deux «preuves », et Russell (dans Russell, 1921, p. 263-270) consacrera un long développement à leur réfutation - en un mot, les deux raisonnements violent les garde-fous

7. Voir Russell, 1903, $\mathbb{S} 54$. Une des raisons qu'a Russell d'introduire la notion de «meaning» est précisément qu'elle permet d'expliquer comment nous pouvons saisir des classes infinies. Penser des «meaning» infinis lui est donc interdit.

8. Russell, 1903: «Et, en ce qui concerne Hegel, il crie au loup si souvent que lorsqu'il tire l'alarme de la contradiction, nous avons fini par rester de marbre. »

9. Van Heijenoort, 1967, p. 101 : «Un tel système infini existe-t-il vraiment dans notre monde de pensées? Sans une preuve logique d'existence, le fait que de tels systèmes ne contiennent aucune contradiction cachée resterait à jamais douteux. D'où le besoin d'une telle preuve.» 
typologiques nous prémunissant contre la contradiction ${ }^{10}$. Le philosophe, suivant en cela les pères de la théorie des ensembles, ajoute donc, dans les Principia, l'axiome de l'infini au stock des principes logiques. Dans Introduction à la philosophie mathématique, Russell se contente de soutenir que le fait que nous ne puissions savoir a priori s'il existe des classes infinies n'autorise pas à affirmer, comme Bradley le fait constamment, que l'infini est contradictoire.

La démarche de Royce ressemble, en son commencement du moins, fortement à celle de Russell. Le philosophe rappelle d'abord les conséquences des thèses de Bradley (Royce, 1901, p. 485):

Les critiques de $\mathrm{M}$. Bradley ont très souvent exprimé leur désapprobation devant la position extrêmement délicate dans laquelle, dans cette théorie, notre pensée finie est laissée. Nous sommes censés définir le Réel comme un système à l'intérieur duquel l'unité et la diversité sont en harmonie. Nous sommes censés concevoir cette réalité comme une "expérience sensible». Et dans l'Expérience absolue, rien de notre variété finie n'est censé être perdu, mais tout est «transmuté ». Et cependant, chaque situation [...] dans laquelle, par un processus intellectuel, ou par un type de conscience médiatisée, nous semblons, nous hommes, avoir réussi à établir une synthèse explicite et une harmonie entre l'Un et le Multiple, est sévèrement rejetée par M. Bradley comme ne fournissant aucune indication sur la manière dont, dans l'Absolu, unité et multiplicité sont unis.

Le philosophe américain concentre alors son attention sur un texte il s'agit de l'appendice Contradiction and Contrariety (Bradley, 1893) dans lequel Bradley expose les conditions que devrait, selon lui, satisfaire une pensée vraie:

Si on demande maintenant ce qui pourrait «satisfaire l'intellect, en supposant que cela puisse être atteint », [Bradley fait la réponse suivante]: «Si les divers [diversities] étaient des aspects complémentaires d'un processus de connexion et de distinction, le processus n'étant pas extérieur aux éléments, ou encore, s'il n'était pas une compulsion étrangère [foreign compulsion] à l'intellect, mais le proprius motus de l'intellect lui-même, le cas serait différent. Chaque aspect serait de lui-même une transition vers l'autre aspect, une transition immédiatement intrinsèque et naturelle à elle-même et à l'intellect. [...] Et la question comment et pourquoi le divers est un et l'un divers perdrait ici sa signification.» Mais M. Bradley (p. 56) est «incapable de trouver [verify] une solution de ce genre».

10. Citons le début du passage (Russell, 1921, p. 263) : «Avant d'analyser de près [l'argument], la première chose à dire est qu'il ressemble à un tour de passe-passe: on pense au prestidigitateur qui sort des choses de son couvre-chef. Le spectateur qui a prêté son couvrechef sait bien qu'il ne contenait pas de lapin, mais il serait bien en peine de dire comment un lapin vivant a pu s'y fourrer. De même, le lecteur qui a un sens robuste de la réalité sentira bien qu'il est impossible de fabriquer une collection infinie à partir d'une collection finie d'individus, même s'il est incapable de trouver la faille dans la construction. » 
Toute la recherche de Royce vise à montrer qu'une solution de ce genre existe pourtant ${ }^{11}$ : l'ensemble des procédures de récurrence, qui traversent l'arithmétique, répond à la description faite ici par Bradley, à condition d'être conçu comme Dedekind le propose. Royce va donc, dans la suite du texte, s'employer à montrer que Was sind und was sollen die Zablen? nous présente le raisonnement arithmétique comme un processus où l'intellect n'a affaire qu'à lui-même et est à lui-même son proprius motus.

Á la différence de Russell, il ne s'agit donc pas seulement, pour Royce, d'utiliser les travaux de Dedekind et de Cantor pour montrer qu'un concept, déclaré contradictoire par Bradley, ne l'est pas, ou ne l'est pas nécessairement. Le philosophe américain veut plus: montrer que la façon dont Dedekind présente l'arithmétique correspond point par point à la description bradleyenne de ce que serait une connaissance de l'Absolu, si elle était possible. Il ne s'agit donc pas seulement pour Royce de dire: Bradley a une vision extrêmement étroite de ce qu'est la pensée - vision que la considération des mathématiques nous permet d'élargir - mais d'affirmer que l'arithmétique, telle qu'elle est exposée dans Dedekind, 1888, réalise exactement ce que Bradley tient pour impossible, et qu'en conséquence une transformation de l'idéalisme "sceptique" de Bradley en un nouvel idéalisme, rationaliste, est non seulement envisageable, mais nécessaire ${ }^{12}$.

\section{La lecture idéaliste de Was sind und was sollen die Zahlen?}

Avant d'en venir au texte de Royce, quelques rappels sur la théorie de Dedekind. Brièvement dit, il s'agit, pour le mathématicien, de définir la structure $\mathbb{N}$ des nombres entiers, à partir de concepts plus fondamentaux, qualifiés de «logiques", la notion d'ensemble [System] et d'application [Abbildung]. S'il n'a rien aujourd'hui d'extraordinaire, le projet est à l'époque inédit, et l'ouvrage de Dedekind mettra du temps à s'imposer ${ }^{13}$. Sur la notion d'ensemble, nous ne dirons rien, si ce n'est qu'un ensemble, pour Dedekind, n'est pas nécessairement fini - si ce n'est également que l'usage de ce concept sera critiqué par Frege ${ }^{14}$. La notion d'application demande en revanche quelques éclaircissements. Une «Abbildung » $\varphi$ du système $S$ est une «loi »

11. N. Griffin a montré qu'un projet similaire animait le premier ouvrage de McTaggart (McTaggart, 1968), manuscrit resté non publié à l'époque, mais qui a été largement diffusé, et qui a notamment eu un impact considérable sur le jeune Russell; voir, pour plus de détails, Griffin, 1991.

12. Royce a reçu une solide formation mathématique. Notons d'ailleurs qu'il développera, à partir de 1900, comme Russell, une théorie générale de l'ordre, basée sur les développements du mathématicien A. B. Kempe, qui va à son tour avoir une influence non négligeable sur la pensée du géomètre américain Veblen; sur ce point, que nous laisserons ici de côté, voir Grattan-Guinness, 2000, p. 371-379.

13. Voir Dugac, 1976, p. 93-96, pour une analyse de la réception.

14. Outre certains reproches techniques, Frege s'objectera à l'approche purement extensionnelle de Dedekind. 
qui associe à chaque élément $s$ de $S$ un élément $\varphi(s)$ de $S$, appelé l'image de $s$. À part la référence à la «loi ", on retrouve bien ici le concept usuel d'application entre ensembles. Dedekind introduit dans la section $3(\mathbb{S} 26)$ la notion d'application injective ou similaire (änlich [oder deutlich] Abbildung) de la façon attendue: $\varphi(s)$ définie sur $S$ est injective (similaire) si à deux éléments quelconques de $S$ correspondent différentes images par $\varphi$. Le mathématicien étend cette notion de similarité aux ensembles eux-mêmes: si $\varphi$ est injective (similaire) sur $S$, alors $\varphi(S)$ et $S$ sont dits semblables. Comme la relation de similarité entre systèmes est transitive, les systèmes similaires ou équipotents forment des classes exclusives les unes des autres (des classes d'équivalence).

Cette machinerie mise en place, Dedekind cherche, dans la section 4, à définir quelles sont les conditions les plus générales garantissant la validité de l'induction mathématique. Pour des raisons de commodité, je ne vais pas suivre l'ordre de son exposition, mais présenter directement la façon dont il introduit $\mathbb{N}$ dans la section $6^{15}$. Une lecture rapide pourrait nous faire croire que Dedekind définit $\mathbb{N}$ comme n'importe quel couple $\langle N, \varphi>$ tel que :

a) $\varphi$ est une application injective.

b) $\varphi(N) \subset N$.

c) 1 est un élément distingué de $N$ qui n'appartient pas à $\varphi(\mathrm{N})$.

Intuitivement, Dedekind définirait, à partir de $N$, un premier ensemble $\varphi(N)$ différent de $N$, puisqu'il ne contient pas l'élément distingué 1 ; puis un second: $\varphi \varphi(N)$, non identique à $N$ parce qu'il ne contient pas $\varphi(1)$; un troisième : $\varphi \varphi \varphi(N)$, non identique à $\varphi \varphi(N)$, parce qu'il ne contient pas $\varphi \varphi(1)$;

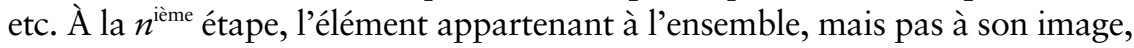
serait le nombre $n$.

Cette première caractérisation de $\mathbb{N}$, on verra bientôt pourquoi, échoue. Mais, bien qu'insuffisante, elle possède déjà des traits remarquables. La relation $\varphi$ s'applique à une classe, non pas à un objet, comme ce sera le cas chez Peano pour la relation de succession. Dans Was sind und was sollen die Zablen? un ensemble est pris pour base, et l'opération indéfiniment réitérée consiste à ôter de l'ensemble un élément, cet élément exclu étant identifié au nombre entier. Le jeu ne consiste pas à engendrer, à partir d'un premier objet, un nombre infini d'éléments, mais au contraire à épuiser un ensemble donné, l'ensemble $N$, en lui ôtant un à un ses éléments. Cela a une conséquence importante: pour que $\langle N, \varphi>$ ait la structure de $\mathbb{N}$, il faut que l'ensemble $N$ soit infini. Si $N$ était fini, alors il n'existerait pas de $\varphi$ injective, telle que $1 \notin \varphi(N)$.

15. Nous reprenons la présentation de Ferreiròs, 1999, p. 222-232, elle-même inspirée par l'ordre d'exposition adopté dans la lettre à Keferstein; mais voir également Dugac, 1976, p. 79-93. 
Mais, quel que soit son intérêt, cette première tentative, on l'a dit, échoue, et ce, même si on fait l'hypothèse que $N$ est infini. Pourquoi ? Parce qu'une telle description s'applique à n'importe quel système infini $S$, qui contient, au-delà de la suite des entiers, un ensemble arbitraire $T$ stable par l'opération $\varphi$. C'est pour éliminer ces "modèles non standard ", qui rendent tout usage du principe d'induction impossible, que Dedekind introduit, dans la section 4 , le concept clé de chaîne d'un système $A$ pour une application $\varphi$ donnée, dénoté par $A_{0}$, où $A$ est une partie quelconque de $N: A_{0}$ est l'intersection de tous les sous-ensembles $K$ de $S$ qui sont tels que $\varphi(K) \subset K$, et qui contiennent $A^{16}$. Dit autrement, $A_{0}$ est le plus petit ensemble clos par $\varphi$ qui contient $A$. Par exemple, l'ensemble $\{3,4,5\}$, sous-ensemble de $N$, n'est pas stable par l'application $\tau(n)=n+1$ - et la chaîne de $\{3,4,5\}$ par $\tau$ est l'ensemble des entiers supérieurs ou égaux à 3 . On a à présent tout en main pour énoncer la condition supplémentaire permettant de caractériser $\mathbb{N}$ complètement:

d) $N$ doit être la chaîne de 1, c'est-à-dire la plus petite chaîne contenant 1 .

Un ensemble $N$ satisfaisant ces quatre conditions est appelé un système simplement infini, et il est isomorphe à $\mathbb{N}$.

Nous n'insistons pas sur ce dernier raisonnement, pourtant essentiel du point de vue mathématique, car il n'intervient pas dans la discussion de Royce $^{17}$. Ce qui en revanche est absolument crucial est le fait déjà évoqué que, pour définir les nombres finis, Dedekind parte de l'infini. Pour le dire de façon grossière, s'il n'y avait que des ensembles finis, s'il n'y avait, par exemple, que des ensembles à deux éléments, alors, non seulement l'entier 3 , mais également l'entier 1, n'existeraient pas - cela, parce que le 1 est, selon Dedekind, essentiellement le premier élément d'une série (simplement) infinie. L'infini est, dans cette perspective, au fondement de l'arithmétique, de la science des nombres finis ${ }^{18}$.

Dans la théorie des ensembles classique, un axiome, l'axiome de l'infini, garantit, nous y avons déjà fait allusion, l'existence d'un ensemble infini. Dedekind, lui, parce qu'il cherche à dériver l'arithmétique des seuls concepts

16. Voir la lettre à Keferstein (Van Heijenoort, 1967, p. 100) : «Que doit-on ajouter aux faits précédents afin de prémunir notre système $S$ contre de tels intrus [...] qui perturbe tout vestige d'ordre $[\ldots]$ ? ?

17. Non pas que Royce ignore l'importance mathématique de la question — le philosophe se montre en réalité étonnamment au fait des différentes possibilités ouvertes par le concept de chaîne (Royce, 1901, p. 519-525). Mais dans sa lecture "métaphysique ", le point n'est pas utilisé; ce qui ne va pas, d'ailleurs, sans poser quelque difficulté (voir infra.).

18. Voir cette caractérisation lapidaire du projet dedekindien par Hilbert (Hilbert, 1922), p. 1121: «Aussi fascinante et éblouissante que soit l'idée de Dedekind consistant à fonder les nombres finis sur l'infini, nous savons en toute certitude aujourd'hui [...] que ce chemin ne peut être parcouru. » Notons que Russell critique Dedekind précisément sur ce point (Russell, 1903, p. 248): «Dans la méthode de Dedekind [...], les propositions concernant les nombres particuliers, comme les propositions générales, requièrent la considération des chaînes. » 
d'ensemble et d'application, souhaite déduire cette existence sans poser de postulat particulier. Cette "déduction", fondamentale pour lui, occupe le célèbre paragraphe 66 de Die Zahlen, très inspiré par Bolzano. Dedekind commence par y donner une définition de l'infini, celle-là même que l'on retrouve chez Cantor et qui sera reprise par Russell: un ensemble $S$ est infini s'il est similaire à une de ses parties propres, c'est-à-dire, si $\varphi$ est injective et si $\varphi(S) \subset S$, avec $\varphi(S) \subsetneq S$. Il construit ensuite un ensemble infini $S$ en considérant ce qu'il nomme "mon monde de pensée " (meine Gedankenwelt), c'est-à-dire l'ensemble des choses qui peuvent devenir objet pour ma pensée. À chaque élément $s$ de cet ensemble, Dedekind associe $\varphi(s)$, «s peut être objet de ma pensée ", qui est, dit-il, un nouvel objet de ma pensée et appartient donc à «meine Gedankenwelt» — on a en conséquence $\varphi(S) \subset S$. D'autre part, il "vérifie » ${ }^{19}$ que $\varphi$ est une application et qu'elle est injective. Il reste, pour montrer que $S$ est infini, à prouver que $\varphi(S)$ est une partie propre de $S$, ce qui est, selon le mathématicien, aisé: mon propre moi, "mein eigenes Ich", est une chose qui peut être objet pour ma pensée, mais qui n'est pas lui-même de la forme $\varphi(s)$. Mon monde de pensée est donc nécessairement un ensemble infini, et aucun axiome spécifique n'est nécessaire pour construire $\mathbb{N}$. Bien entendu, cette "preuve " tombe sous le coup de la critique russellienne: mon monde de pensée est un objet contradictoire, qui viole les réquisits typologiques ${ }^{20}$. Mais ce qui importe ici est simplement de rappeler que cette "démonstration" occupe une position centrale dans l'ensemble du dispositif dedekindien.

Qu'est-ce que Royce retient de Die Zablen? Essentiellement deux choses, que le philosophe lie l'une à l'autre: la définition d'un ensemble infini d'abord, plus particulièrement la condition de "réflexivité ", que Royce rebaptise "self-representativity": $\varphi$ est injective, $\varphi(S) \subset S$ et $\varphi(S) \subsetneq S$; l'usage de "mein eigenes Ich» et du "Gedankenwelt» dans la preuve de l'existence de l'infini, ensuite. Mais avant de parler du fond, soulignons le travail, stylistique, de reformulation effectué Royce. Le philosophe consacre en effet une longue partie de son texte à traduire, en des termes non mathématiques, la condition de "self-representavity». L'illustration qu'il propose est restée, à juste titre, célèbre. C'est celle de la carte de l'Angleterre faite sur le territoire de l'Angleterre. Je cite Borges, se référant lui-même à Royce (Borges, 1957, p. 85$)^{21}$ :

Josiah Royce, dans le premier volume de World and the Individual (1899), a [écrit]: "Imaginons qu'une portion du sol de l'Angleterre ait été parfaitement nivelée, et qu'un cartographe y trace une carte de l'Angleterre. L'ouvrage est parfait; il n'est pas un détail du sol de l'Angleterre, si réduit soit-il, qui ne soit enregistré sur la carte; tout s'y retrouve. Cette carte, dans ce cas, doit contenir

19. Voir Dugac, 1976, p. 88-89.

20. Pour une analyse des hésitations et des réactions de Dedekind, voir Dugac, 1976, p. 89.

21. Russell mentionne l'illustration dans Russell, 1921, p. 164. 
une carte de la carte, qui doit contenir une carte de la carte de la carte, et ainsi jusqu'à l'infini. »

$S$ est ici le territoire de l'Angleterre, et $\varphi$, l'application injective qui envoie chaque point du territoire anglais sur un point de la carte. Comme la carte est réalisée sur une portion seulement du territoire, on a à la fois $\varphi(S) \subset S$ (la carte est dans le territoire) et $\varphi(S) \subsetneq S$ (la carte ne recouvre pas tout le territoire). Le dispositif, simple et maîtrisé, représente de façon très suggestive l'ensemble des éléments que l'on trouve dans la condition de réflexivité ${ }^{22}$.

Royce n'est pas le seul à avoir imaginé des scenarii destinés à illustrer la définition dedekindienne de l'infini. Russell évoque ainsi dans les Principles, le paradoxe de Tristram Shandy ${ }^{23}$, qui s'attriste d'avoir mis un à à écrire l'histoire des vingt-quatre premières heures de sa vie, et de ne pouvoir, à ce rythme, achever sa biographie. S'il vivait infiniment longtemps, Tristram Shandy n'aurait aucune raison de se plaindre, affirme Russell, car alors il pourrait, non pas évidemment terminer son histoire, mais ne laisser aucun jour de son existence non raconté dans sa biographie. De Borges aux auteurs de science-fiction contemporains ${ }^{24}$, les reprises de ces procédures de mise en abîme sont légion ${ }^{25}$.

Mais ce changement de registre ne doit pas, dans le cas de Royce, être traité simplement comme une élégante variation stylistique, destinée à habiller un nouveau et fascinant concept mathématique. Il semble qu'elle prépare à beaucoup plus, à un rapatriement de la notion d'infini dans le champ de la métaphysique. Royce ne vulgarise pas, à proprement parler, le texte de Dedekind - le lecteur qu'il vise n'est pas «l'honnête homme", mais Bradley lui-même et ses disciples. Ainsi, la notion d'application injective (et l'idée est peut-être déjà présente chez Dedekind quand il emploie le terme "Abbil$d u n g$ ») est spontanément conçue par Royce comme la mise en forme du

22. Royce en joue d'ailleurs - retrouvant des intuitions qui remontent à la découverte grecque des irrationnels (voir notamment la construction des pentagones enchâssés les uns dans les autres, qui «illustre» l'irrationalité du nombre d'or), il affirme que la suite formée des ouverts correspondant aux cartes enchâssées converge vers un point et demande comment varie ce point en fonction de la position de la première carte.

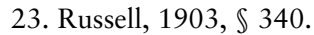

24. Nous pensons en particulier à Philippe K. Dick.

25. Dans les diverses exploitations littéraires du schéma dedekindien, on retrouve d'ailleurs fréquemment la même interprétation : $S$ est très souvent assimilée à la vie et ses événements, $\varphi(S)$ au livre et à son écriture. Citons la belle conclusion de Magies partielles $d u$ "Quichotte» qui propose une analyse des raisons du succès de ce dispositif (Borges, 1957, p. 85): «Pourquoi sommes-nous inquiets que la carte soit incluse dans la carte et les mille et une nuits dans le livre des Mille et une nuits ? [...] Je crois en avoir trouvé la cause: de telles inventions suggèrent que si les personnages d'une fiction peuvent être lecteurs ou spectateurs, nous, leurs lecteurs ou leurs spectateurs, pouvons être des personnages fictifs. En 1833, Carlyle a noté que l'histoire universelle est un livre sacré, infini, que tous les hommes écrivent et lisent et tâchent de comprendre, et où, aussi, on écrit sur eux.» 
concept postcartésien d'idée adéquate, dont l'ordre et la disposition entre les parties préservent l'ordre et la disposition des parties de l'objet représenté $^{26}$. La relation entre la carte géographique et le territoire est d'ailleurs utilisée par Leibniz pour expliquer ce qu'est une expression «fondée dans la nature " (à quoi Leibniz oppose les représentations conventionnelles, linguistiques) ${ }^{27}$. Dans le dispositif de Royce, la définition positive de l'infini se présente comme une extension du concept de représentation adéquate: un système auto-représentatif n'est en effet rien d'autre qu'une idée adéquate qui aurait comme propriété particulière d'être une partie de l'objet qu'elle représente ${ }^{28}$.

Mais, dans la pensée de Royce, c'est surtout par un autre mouvement que s'effectue la rencontre entre mathématiques et métaphysique. Dans son traité, Dedekind séparait de façon nette la définition du système infini de la preuve qu'il existe un tel système. Chez Royce, cette distinction s'estompe. Le Soi ou le monde de mes pensées n'est pas seulement, dans The One, the Many, and the Infinite, un exemple d'ensemble infini; il est le noyau à partir duquel se développe la structure des ensembles «self-representative». Ainsi Royce écrit-il (Royce, 1901, p. 513):

L'usage réellement très profond que fait Dedekind du mein Gedankenwelt comme d'un exemple paradigmatique de l'infini suggère une intéressante relation entre le concept de Soi et celui de la simple forme mathématique appelée la série numérique - une relation sur laquelle nous reviendrons bientôt.

Comme l'emploi de «suggérer » l'indique, Royce est conscient ici de pousser le texte au-delà de son sens originel. Mais, nous allons maintenant le voir, toute son intervention tient précisément dans cette distorsion méditée de la présentation théorique de Dedekind.

26. Royce, 1901, p. 509: «Ces images [de $S$ par l'application injective] sont toutes distinctes, de telle manière que les différents éléments ont toujours différents représentants. » Il est bien question ici du concept classique, disons leibnizien. Royce ne cite cependant pas Leibniz; il se réfère par contre constamment à Spinoza, non pas vraiment d'ailleurs à sa théorie de l'idée adéquate, mais plutôt à sa doctrine de l'idée de l'idée; on verra bientôt pourquoi.

27. Voir le fragment Qu'est-ce qu'une idée? in Leibniz, 1998, p. 446: "On voit aussi que, parmi les expressions, certaines ont un fondement dans la nature, et les autres sont en partie au moins fondées arbitrairement, comme le sont les expressions produites au moyen de sons ou de caractères. Quant à celles qui sont fondées dans la nature, elles postulent ou bien une certaine similitude, comme celle qui existe entre un grand cercle et un petit, ou entre une région et la carte géographique de la région; ou en tout cas une connexion comme celle qui existe entre le cercle et l'ellipse qui le représente optiquement...»

28. Royce définit d'abord la représentation distincte et en vient, seulement dans un second temps, à l'idée de système auto-représentatif (Royce, 1901, p. 504): «Essayons de construire une carte qui sera, dans ce sens [celui d'adéquat], parfaite, mais dont le dessin sera soumis à une condition spécifique. Il semblerait que, si nos capacités de dessinateur de carte étaient parfaites, nous pourrions dessiner notre carte là où nous le souhaitons. Choisissons alors, pour une fois, de la dessiner dans et sur une partie de la surface de la région même à cartographier. À quel résultat conduirait la réalisation de ce projet?» 
La seconde moitié du traité de Royce est en effet consacrée à l'examen des rapports de trois systèmes infinis: le système numérique, le Soi (Self ) et l'Être (Being). Nous allons ignorer complètement tout ce qui relève de l'Être ${ }^{29}$ pour nous concentrer sur les relations entre système numérique et Soi. En un mot, Royce voit dans la référence que Dedekind fait à la structure de "mein Gedankenwelt» un prolongement de la théorie hégélienne et fichtéenne du Soi (Royce, 1901, p. 526):

Ayant considéré l'auto-représentation essentiellement dans l'abstrait, nous pouvons en venir maintenant à d'autres illustrations des systèmes relationnels auto-représentatifs.

En nous lançant dans une telle tâche, nous pourrons, c'est certain, pour la première fois dans cette discussion, énoncer la source logique précise du bon ordre [the good order] du système numérique, dont le caractère auto-représentatif [...] est simplement dû au fait que la série des nombres est une image purement abstraite, pour ainsi dire un squelette nu et desséché, du système relationnel qui caractérise un Soi idéalement complet. Cette observation, dans la forme présente, ne peut pas être imputée à Hegel, bien que son analyse et la doctrine fichtéenne du Soi implique une théorie dont le développement devrait selon toute apparence prendre cette forme plus moderne.

Suit un développement sur le mépris affiché par Hegel et Fichte à l'égard des mathématiques, qui ne vise pas, comme c'est le cas chez Russell, à disqualifier leur métaphysique, mais au contraire à montrer que cette attitude les a empêchés de développer toutes les implications de leurs intuitions. C'est à Dedekind, explique Royce, que l'on doit la redécouverte d'un lien déjà virtuellement présent chez Hegel et Fichte: "L'observation susdite concernant le parallélisme entre la structure de la série des nombres et le squelette nu du Soi idéal est plutôt due à Dedekind qu'aux philosophes idéalistes $^{30}$.»

En quoi Fichte et Hegel anticiperaient-ils le parallèle entre Soi et série numérique? Le lien est, selon Royce, fourni par le concept de système relationnel auto-représentatif, c'est-à-dire par la condition $\varphi$ est injective, $\varphi(S) \subset S$ et $\varphi(S) \subsetneq S$. Ce serait cette auto-réflexivité que Hegel et Fichte auraient, selon Royce, mise au fondement du concept de Soi. Si le philosophe américain ne renvoie précisément à aucun texte de Hegel ou de Fichte, il n'est pas difficile de reconstituer son cheminement. Citons un passage célèbre de Fichte (Fichte, 1964, p. 128):

Dans la mesure où le Moi se pose comme infini, son activité (de position) s'applique au Moi lui-même et non pas à quelque chose d'autre qu'au Moi. Toute

29. Le but du philosophe américain semble être de définir le «Being» comme un pur individu et une pure volonté, et son propos, souvent confus, s'éloigne heureusement ici à la fois de la critique de Bradley et du texte de Dedekind.

30. Royce, 1901, p. 527. 
son activité s'applique au Moi, et cette activité est le fondement de la sphère de l'être en totalité. Il s'ensuit que le Moi est infini pour autant que son activité se réfléchit en elle-même et en ce sens que son activité est elle aussi infinie, étant donné que son produit, le Moi, est infini. [...] Seule la pure activité du Moi, seul le Moi pur sont infinis. L'activité pure est celle qui n'a pas d'objet, mais qui se réfléchit en elle-même.

Le Moi est essentiellement, selon Fichte, conscient de soi : il est essentiellement l'activité de se réfléchir lui-même en lui-même ${ }^{31}$. Et c'est la caractérisation du Soi comme être se rapportant négativement à lui-même, se réfléchissant en lui-même, que Royce identifierait avec le concept de système auto-représentatif. On pourrait ainsi proposer la «traduction » suivante: $S$ serait le Moi ou le monde des pensées; $\varphi(S)$ jouerait le rôle de l'activité réfléchissante qui, à chaque élément du monde des pensées, associe sa représentation, et $S \backslash \varphi(S)$ (le complément de $\varphi(S)$ dans $S$ ) correspondrait à l'Être ou au monde qui fait face au Moi (aux objets donnés que l'activité de représentation prend pour objet). D'un côté, $\varphi(S)$ est l'image de $S$ par $\varphi$, c'est-àdire que le sous-ensemble $\varphi(S)$ représente $S$ - le Moi reflète l'ensemble de tous les objets, qui, en ce sens, sont tous des objets pour un Moi; d'un autre, on a $\varphi(S) \subset S$ et $\varphi(S) \subsetneq S$, c'est-à-dire que $\varphi(S)$ n'est qu'une partie de $S-$ le Moi et ses pensées sont eux-mêmes des objets parmi d'autres, dont une représentation peut être formée. Autrement dit, $S$ serait tout à la fois un être et l'opération de se différencier en une image de soi qui n'est pas soi ${ }^{32}$. L'acte de réflexion, que Fichte, puis Hegel, mettent au centre de la structure du Soi, jouerait donc ici le rôle de l' "Abbildung » similaire $\varphi$ : le Moi apparấtrait à la fois comme une chose parmi d'autres $(\varphi(S))$, et comme l'être luimême, que je ne peux nier qu'en me niant moi-même $(S)$.

En ce qui concerne le lien tissé entre la définition "abstraite» d'un ensemble infini et le concept de Soi, deux choses méritent d'être notées. En premier lieu, même si la notion de Soi est "concrète", au sens où elle illustre et exemplifie le concept de système auto-représentatif, Royce distingue explicitement le Soi idéal, pur, du Soi psychologique. La critique, adressée notamment par Russell à Dedekind, selon laquelle nous n'avons aucune expérience de l'idée de l'idée, et ainsi de suite, n'est pas recevable, car le Self dont il est question ici n'est pas le Soi empirique, mais le Soi objectif, ou plutôt le concept de Soi, tel qu'il est développé par Fichte et

31.Voir également cet autre passage (Fichte, 1964, p. 21): «On entend souvent poser la question: qu'étais-je avant de parvenir à la conscience de soi ? La réponse naturelle est la suivante: je n'étais absolument pas; en effet je n'étais pas Moi. Le Moi n'est que dans la mesure où il est conscient de soi. » Je remercie G. Rametta de m'avoir aiguillé sur ces textes.

32. Cf. par exemple ce passage (Royce, 1901, p. 524), où Royce, commentant l'argument de Dedekind, mobilise spontanément la terminologie du Soi: «Mais puisque $[\varphi(S)]$ représente la totalité de la série des nombres originale, elle doit contenir, comme partie de soi-même, une représentation de son propre soi (a representation of its own self) comme c'est le cas dans la série des nombres.» 
Hegel. Le second point sur lequel Royce met l'accent est que la véritable notion de totalité n'est pas, contrairement à ce que croit Bradley, donnée dans l'expérience intuitive immédiate, mais fournie par la notion de Soi dont Dedekind construit le squelette formel. L'essence de la totalité est enveloppée dans l'idée de complétude, c'est-à-dire d'auto-représentation: un système incomplet est un système auquel il manque la représentation de soi-même. Ainsi, comme Royce l'explique (Royce, 1901, p. 553):

"L'Univers ", c'est-à-dire le véritable tout, en tant qu'[il est] Sujet-Objet, contient une image complète et parfaite, ou une conception de soi-même. Il est, en conséquence, dans sa structure, tout à la fois Un, en tant que système unique, et aussi une Kette infinie. Sa forme est celle d'un Soi.

Nous avons désormais en main toutes les cartes pour comprendre la critique que Royce adresse à Bradley. Die Zablen permet de répondre affirmativement à la question concernant l'existence d'un système satisfaisant les demandes de l'intellect (Royce, 1901, p. 534):

L'auto-représentation [Self-representation] [...] n'est pas simplement, pour ainsi dire, la propriété ou l'accident de la série des nombres mais est, logiquement parlant, son principe génétique. [...] Ce que nous remarquons ici est que la constitution de la série des nombres, avec toutes ses conséquences, est explicitement définie comme la forme d'un Soi complet, considérée bien entendu dans son ébauche la plus simple et la plus abstraite. Ici enfin, l'Intellect, «de par son propre mouvement ", "lui-même par lui-même ", définit ce qui, dans notre expérience temporelle, qu'elle soit sensible ou intellectuelle, ne se trouve bien entendu nulle part comme donné, à savoir un système auto-représentatif d'objets, parallèle dans sa structure avec ce que serait la structure du Gedankenwelt s'il était le Welt d'une pensée complètement consciente d'elle-même, telle qu'aucun de ses actes n'échouerait à être un de ses objets intellectuels propres.

Selon Royce, le système auto-représentatif satisfait complètement aux exigences de l'intellect, telles que Bradley lui-même les avait formulées dans l'appendice Contradiction and Contrariety - ce concept est donc ce qui permet d'échapper au scepticisme et de substituer à l'idéalisme "sceptique » de Bradley un nouvel idéalisme, rationaliste dans son essence (Royce, 1901, p. 537):

Notre pensée recherche son propre travail comme son objet. C'est l'essence même de cet effort que de laisser l'intellect exprimer son propre mouvement [self-movement]. Mais faire de son propre travail son objet, observer de nouveau ce qui a été accompli, c'est simplement rétablir, comme un fait encore à connaître, le processus même dont le premier résultat est observé lorsque l'intellect contemple l'acte [deed] qui vient d'être accompli. [...] Ce qui nous intéresse est la structure positive de la totalité du monde intellectuel. Nous avons trouvé cette structure. C'est la structure d'un système auto-représentatif [...]. 
Non seulement Royce lie, en détournant la lettre du texte de Dedekind, le concept de Soi et celui d'infini, mais il fonde sur cette idée une critique de la version bradleyienne de l'idéalisme. Le Soi est, selon Royce, la véritable forme de l'Absolu, et l'Absolu est donc une totalité dont l'intellect peut saisir la structure abstraite (Royce, 1901, p. 553-554): "Aucune philosophie qui ignore complètement ce fait élémentaire [c.-à-d. que tout ce qui est est un système auto-représentatif] ne peut être appelée rationnelle. »

\section{L'interprétation de Royce est-elle historiquement pertinente?}

Comme nous l'avons déjà dit, Royce ne s'arrête pas là. Le philosophe américain, non satisfait par le lien établi entre subjectivité et arithmétique, tente de prouver que l'Être lui-même est un système auto-représentatif - et il développe pour ce faire une théorie assez confuse de l'individualité et de la volonté, dont le lien avec l'opuscule de Dedekind est très lâche. Au lieu de continuer à suivre Royce dans ce qui nous semble être une impasse, nous allons à présent nous concentrer sur le rapport établi entre la métaphysique fichtéo-hégélienne du Soi et le $\mathbb{} 66$ de Dedekind, 1888. La lecture «métaphysique » que le philosophe américain fait du traité de Dedekind est-elle historiquement fondée?

Royce, répétons-le, ne vise pas à décrire fidèlement le raisonnement de Dedekind, mais à l'exploiter dans un cadre qui n'est pas celui des mathématiques. Le philosophe californien souligne lui-même qu'il prolonge ce qui n'est qu'une "suggestion "; en confondant volontairement les deux moments de la définition d'un système infini et de la preuve d'existence, Royce sait qu'il va au-delà de la lettre même de Die Zablen ${ }^{33}$. Mais malgré ces distorsions, assumées, n'y a-t-il pas dans l'usage que fait Royce des travaux d'un mathématicien dont la réputation de sobriété en matière de commentaires métaphysiques n'est plus à faire quelque chose de pertinent et de profondément juste ? Nous aimerions, dans ce qui suit, pousser l'hypothèse de Royce au delà d'elle-même, et explorer la possibilité d'un rapport entre les propos de Dedekind et l'idéalisme de Fichte et de Hegel.

Selon toute vraisemblance, c'est au $\mathbb{\$} 13$ des Paradoxes de l'Infini, également évoqué par Russell, que Dedekind reprend l'idée de sa preuve. Bolzano fonde sa démonstration sur la possibilité, à partir d'une proposition donnée $p$, de former une autre " $p$ est vrai », et d'amorcer ainsi une

33. Royce fonde son interprétation de Die Zablen sur le paragraphe 66. De ce point de vue, cette interprétation est diamétralement opposée à celle de Dugac pour qui (Dugac, 1976, p. 88-89): "[Le théorème 66] est le seul théorème de Dedekind dont la "démonstration » ne cadre pas avec la pensée mathématique dedekindienne. [...] On reste quand même perplexe devant cette démonstration, dont le caractère mathématique n'est pas évident, teintée comme elle est de philosophie et même de psychologie! » Royce, s'il oppose métaphysique et psychologie, et s'il prend comme point de départ ce qui doit être considéré, selon Dugac, comme une anomalie, ne dit finalement pas autre chose. 
régression à l'infini ${ }^{34}$. Chez Bolzano, le syntagme "proposition» désigne, non pas un jugement, c.-à-d. le résultat d'une synthèse subjective, mais un objet réel, indépendant de toute activité mentale. Or, sur ce point, Dedekind s'éloigne de son prédécesseur, puisque le monde des pensées auquel il fait allusion au $\mathbb{S} 66$ est explicitement celui d'un Moi. Cet infléchissement apporte de l'eau au moulin de Royce, car rien, sur le plan technique, n'exige que les vérités en soi cèdent la place au "Gedankenwelt». Il semblerait donc que ce soit pour des raisons épistémologiques globales, qui tiennent à l'approche que le mathématicien développe de sa discipline, que Dedekind modifie la présentation de Bolzano, en introduisant, dans le $\mathbb{S} 66$, une référence à un sujet.

Pour tenter d'en comprendre les raisons, nous allons distinguer deux niveaux. Le premier sera celui des influences: cette apparition du Moi dans un traité de mathématique est-il l'écho de discussions philosophiques auxquelles Dedekind aurait pris part, ou au moins dont il aurait eu connaissance? Peut-on attester l'existence d'un lien entre les théories de Fichte et de Hegel, par exemple, et celles de Dedekind? Le second niveau est celui du contexte dans lequel le mathématicien publie son traité: à qui est adressé Dedekind, 1888, et à quoi Dedekind veut-il s'opposer ? Pourquoi Dedekind tient-il à sa preuve de l'existence de l'infini; qui vise-t-elle ${ }^{35}$ ?

Que ce soit dans les textes ou dans la correspondance publiée de Dedekind, rien ne laisse entrevoir un intérêt particulier de Dedekind pour la théorie fichtéenne ou hégélienne du Soi. Les principaux commentateurs de l'œuvre de Dedekind ne font pas non plus mention d'une quelconque influence idéaliste à Göttingen - l'accent est bien plutôt mis sur le rôle prépondérant qu'y joue la pensée de Herbart et de ses disciples, c'est-à-dire sur l'influence d'une forme d'empirisme, fortement lié à la psychologie naissante, et explicitement opposé à l'idéalisme ${ }^{36}$. C'est cependant en allant voir d'un peu plus près les textes d'Hermann Lotze, un des représentants de la

34. Citons le passage central, dans la traduction de H. Sinaceur (Bolzano, 1993, p. 7172): «Comme il est facile de le voir, l'ensemble des propositions et vérités en soi est infini. Si nous considérons, en effet, une vérité quelconque, par exemple la proposition: "Il y a en général des vérités", ou toute autre proposition que je désigne par $A$, nous remarquons que la proposition: " $A$ est vrai" est différente de $A$ elle-même; celle-ci a bien évidemment un tout autre sujet que celle-là, le sujet de la proposition étant la proposition $A$ tout entière. Désignons par $B$ cette deuxième proposition: " $A$ est vraie", et réitérons le procédé de dérivation qui nous a déjà donné $B$ à partir de $A$, nous obtiendrons une troisième proposition à partir de $B$, et ainsi de suite indéfiniment. »

35. Il y aurait une dernière strate à examiner : celle du rapport entre la lecture de Royce et les interprétations contemporaines de Die Zablen. Le traité de Dedekind est aujourd'hui une sorte d'Annapurna de la philosophie des mathématiques: tous les grands courants présentent leur interprétation d'un texte qui semble encore supporter des lectures des plus contradictoires. Dans ce cadre, qui est aussi un champ de bataille, où pourrait-on situer l'interprétation proposée par Royce ? Nous dirons quelques mots à ce sujet à la fin de la section.

36. Sur le lien entre Herbart et Riemann, voir notamment Ferreiròs, 1999, p. 241-247. 
nouvelle philosophie "scientifique ", représentant à plusieurs égards très singulier, que l'on peut trouver de quoi alimenter la thèse de Royce. H. Lotze fut professeur de mathématiques, de physique et philosophie à Göttingen. Dedekind connaissait donc nécessairement sa pensée. On trouve d'ailleurs, dans le Dedekind Nachlass conservé à Göttingen, des notes prises de la main de Dedekind d'un cours de Lotze prononcé en $1852^{37}$. Dans sa Métaphysique de 1841, Lotze critique le tour selon lui excessivement empiriste qu'Herbart a imprimé à la pensée et prône de façon très claire, dans son introduction, un retour vers l'idéalisme objectif et la métaphysique. Or plusieurs indices semblent montrer que, dans le $\mathbb{S} 66$, c'est aux thèses de Lotze que Dedekind se réfère.

En premier lieu, la notion de "Gedankenwelt» joue un rôle fondamental dans la critique que Lotze ${ }^{38}$ fait de Herbart (Lotze, 1883, p. 496):

Toute comparaison de deux idées, qui finit par trouver que le contenu en est égal ou inégal, suppose l'unité complètement indivisible de ce qui exécute cet acte de comparaison: ce doit être tout simplement cela même qui a l'idée de $a$, puis celle de $b$, et qui en même temps s'aperçoit du genre et de la grandeur de la différence qu'il y a entre les deux idées. Ensuite les divers actes d'une telle comparaison, d'un tel rapprochement, deviennent à leur tour des termes dont le rapport mutuel est signalé à la conscience par une nouvelle action comparative; ainsi se construit en nous le monde entier de nos pensées, non comme simple existence simultanée ou comme succession d'idées diverses, mais partout pénétré de cette action unitaire qui, par les relations qu'elle établit entre les divers termes, les ordonne et les maintient ensemble. C'est là ce que nous entendons par l'unité de la conscience, et ce que nous regardons comme un titre suffisant pour autoriser l'hypothèse d'une âme indivisible [je souligne].

Bien entendu, il est possible que cette expression de «monde des pensées» se retrouve ailleurs à la même époque; mais il est remarquable que l'acception du terme soit ici technique: que les pensées forment monde, qu'elles soient unies dans une conscience une, c'est, selon Lotze, à la fois ce que Herbart et ses disciples n'ont pas vu, et ce qui demeure fondamentalement juste dans la théorie fichtéenne et hégélienne de la conscience ${ }^{39}$.

37. Le texte est intitulé Geschichte der neueren deutchen Philosophie, et son contenu semble partiellement coïncider avec Lotze, 1882. Je remercie J. Ferreirós de m'avoir indiqué l'existence du manuscrit.

38. Je remercie A. Pettoello et C. Maigné de me l'avoir indiqué.

39. Il y aurait ici beaucoup à dire sur le concept de comparaison. Un passage du Onzième supplément aux conférences de Dirichlet sur la théorie des nombres, reproduit dans Ewald, 1996, p. 834, suggère un lien entre la comparaison et l' «Abbildung» de Dedekind: "Aucune pensée d'aucun genre n'est possible sans le pouvoir de l'esprit à comparer une chose $a$ avec une chose $a^{\prime}$, ou de relier $a$ à $a^{\prime}$, ou de permettre à $a$ de correspondre à $a^{\prime}$ - et la science entière des nombres repose également sur cette capacité. L'élaboration de cette idée a été [...] publiée dans mon article "Was sind und was sollen die Zablen?" " 
D'autre part, et peut-être surtout, on trouve, toujours dans la troisième partie de la Métaphysique, des passages proches du contenu du $\mathbb{\$} 66$. Je cite un extrait, particulièrement intéressant, dans lequel, contre Herbart, Lotze défend l'idée que les rapports entre idées ne résultent pas de la simple association, mais d'une réaction réflexive de l'âme à ses idées (Lotze, 1883, p. 559):

Il se comprend de soi-même que, d'après [ma] conception, tout état quelconque de l'âme, ou toute série d'actes intellectuels qui ont été appliqués à différentes idées pour les comparer et les juger, peut également devenir pour l'âme une nouvelle excitation, l'objet d'une réflexion plus haute encore; mais il y aurait de l'enfantillage à vouloir compter des réactions de troisième ordre et de quatrième ordre, tant qu'on ne pourrait, au moyen d'une psychologie détaillée, dont ce n'est pas ici la place, indiquer exactement, dans l'observation intérieure, les phénomènes qui justifieraient l'admission de divers degrés de prééminence. Métaphysiquement, on ne va pas, avec cela, plus loin qu'avec la simple reconnaissance de ce caractère de l'âme qui fait qu'elle est non pas simplement une enceinte pour la foule des états intérieurs, mais le sol vivant qui, par chaque création momentanée dont il a déterminé l'épanouissement, a, en même temps, fait naître en soi de nouvelles conditions pour la production de créations plus élevées [je souligne].

Si la réflexivité, qui donne en droit naissance à une régression à l'infini n'a en elle-même aucun ancrage introspectif réel, il n'en demeure pas moins vrai que le lien entre ce concept et celui de Moi doit être, selon Lotze, réaffirmé. L'idée, qui sera d'une certaine façon reprise par Dedekind, de vouloir «compter des réactions de troisième ordre et de quatrième ordre " est, certes, d'un point de vue psychologique, un enfantillage - mais, d'un point de vue métaphysique, cet accent mis sur la réflexivité interne à la structure du Soi n'est qu'une manière d'affirmer que l'âme n'est "pas simplement une enceinte pour la foule des états intérieurs, mais le sol vivant qui, par chaque création momentanée [...] fait naître en soi de nouvelles conditions pour la production de créations plus élevées». Lotze conjoint, dans ce texte, l'ensemble des éléments rassemblés par Royce: une référence claire au concept idéaliste du Soi et à la réflexivité, une critique de la conception psychologisante du Moi, une amorce de récurrence et un lien avec les entiers - auxquels s'ajoute, de surcroît, une terminologie qui rappelle celle employée par Dedekind (l'esprit comme «sol vivant", qui produit des "créations» toujours plus élevées). $\mathrm{Si}$ l'hypothèse d'un lien direct entre Fichte, Hegel et Dedekind ne paraît pas pouvoir être attestée, un lien indirect, passant par Lotze, pourrait exister: l'analyse de Royce ne serait donc pas, de ce point de vue, absolument sans fondement historique. Nous allons renforcer cette première conclusion par l'examen du contexte mathématique et épistémologique dans lequel écrit Dedekind.

Dans les présentations de Was sind und was sollen die Zablen?, deux lignes problématiques sont habituellement évoquées: d'une part, l'opposition à Kant et à toute forme d'intuition du numérique, commune à Dedekind et Frege; d'autre part, la position de Dedekind vis-à-vis de l'infini actuel et du 
finitisme de Kronecker. Il semble que la lecture de Royce éclaire sous un nouveau jour ces deux mises en perspective.

L'interprétation de Royce permet en premier lieu d'élaborer l'articulation à Kant. Fonder l'arithmétique sur la logique ne signifierait pas seulement, selon Dedekind lu par Royce, se défaire de l'idée que le concept de progression ne prend son sens que grâce à l'intuition formelle du temps - fonder l'arithmétique sur la logique, ce serait contester jusqu'au rôle que Kant fait jouer à la temporalité dans la doctrine du Moi. La temporalité est en effet, selon Kant, la raison pour laquelle le Moi n'est jamais saisi comme chose en soi, mais toujours seulement comme phénomène. Citons un extrait de l'Anthropologie (Kant, 1988, p. 28):

Je suis, en tant qu'être pensant, un seul sujet, et le même que le moi en tant qu'être sensible; mais en tant qu'objet de l'intuition empirique interne, c'est-àdire dans la mesure où je suis affecté intérieurement par des sensations dans le temps, $[. .$.$] je me connais seulement comme je m'apparais à moi-même, non$ pas comme chose en soi [je souligne].

Le temps joue chez Kant un double rôle: rôle positif, en tant que la forme de l'intuition interne fournit à l'arithmétique sa matière propre; rôle négatif, dans la mesure où la temporalité, qui reste une modalité du sens interne, empêche de saisir le Moi autrement que comme phénomène. L'intérêt de Royce est de ne pas disjoindre les deux choses. La critique du rôle que joue la temporalité dans l'arithmétique est immédiatement liée chez lui à une description de la structure atemporelle du Soi. Ou, dit autrement, l'approche logique de la structure $\mathbb{N}$ est immédiatement conçue comme le prolongement naturel d'une conception logique, fichtéo-hégélienne du Soi, qui le détache de tout rapport à la temporalité: le fait contingent que nous, hommes, comptions, sentions, pensions dans le temps, ne signifie pas que la structure des entiers et celle du Moi soient liées à (et voilées par) la temporalité (Royce, 1901, p. 582):

Déterminer si cet élément ou cet autre appartient à la Kette, peut nous prendre ou non un long moment. Il implique pour nous la succession, des processus de décompte, et beaucoup de choses du même genre. Cela, cependant, est dû à notre fortune d'observateur humain [is due to your fortune as a human observer].

Le concept de système auto-représentatif permet de penser à la fois la structure non phénoménale, logique, des entiers, et la structure non phénoménale, logique, de l'esprit: chez Royce, le projet dedekindien de logicisation de l'arithmétique est conçu comme l'élément d'un ensemble plus vaste, visant une redéfinition du concept du Soi.

En ce qui concerne maintenant l'autre type de contextualisation, celle consistant à interroger la place de Dedekind dans le débat sur l'infini actuel opposant Kronecker et Cantor, là encore, l'interprétation proposée par Royce permet d'ouvrir de nouvelles perspectives. J. Ferreirós affirme que si la défense de l'infini actuel est incontestablement une réponse aux critiques 
finitistes et constructivistes de Kronecker, la façon dont Dedekind formule sa thèse l'éloigne des conceptions réalistes cantoriennes, car, là comme ailleurs, le mathématicien de Göttingen souligne le rôle de l'activité humaine et de la libre création (freie Schöpfung). Dedekind chercherait, dans Die Zablen, une voie médiane entre le finitisme kroneckerien et le platonisme cantorien (Ferreiròs, 1999, p. 244-245):

Dedekind n'était manifestement pas un platonicien, mais il n'était pas non plus un empiriste: les objets mathématiques n'existent absolument pas hors de notre esprit, mais ils ne sont pas non plus le simple résultat de nos perceptions ou de l'expérience du monde physique. [Dedekind] fut toujours convaincu que les objets mathématiques et les concepts sont des créations de l'esprit humain [...]. Ce n'est pas par hasard que Zahlen a pour motto: "L'homme toujours arithmétise ", où l'homme remplace le Dieu de ses prédécesseurs. Pour cette raison, il avait un certain biais "nominaliste ", au sens où il évitait de faire référence à des objets abstraits subsistant par soi [...].

Si le diagnostic du commentateur nous semble correct, la référence au «nominalisme », dans la dernière phrase, manque de précision: à quel sens du nominalisme Ferreirós fait-il appel ici ? Comment caractériser la position assumée par Dedekind de façon plus précise?

L'interprétation proposée par Royce suggère une réponse. Ce ne serait pas sur le Soi psychologique, mais sur le Soi idéal, le «squelette nu et desséché [d'un] Soi idéalement complet ", dit Royce, que Dedekind s'appuierait. La distinction nous permettrait de saisir la nature de l'opposition à Kronecker: Dedekind défend l'idée d'un infini actuel et rejette les limites finitistes comme simplement psychologiques (le Moi n'est pas une «enceinte pour la foule des états intérieurs ", disait Lotze) - tout en évitant de poser l'existence d'objets en soi. Ce serait en effet la structure d'un être très particulier, le Soi, qui serait en elle-même infinie. Selon cette lecture, le $\mathbb{} 66$ ne fournirait pas simplement une preuve d'existence d'un système infini - il dévoilerait le sens et la portée véritable du projet d'arithmétisation: manifester le lien essentiel entre les mathématiques et la structure de l'esprit. Ce serait bien (contre Cantor) «l'homme» qui «toujours arithmétise»: la théorie des nombres doit être fondée sur la structure de l'esprit humain. En même temps, l'esprit dont il est question ne serait pas (contre Kronecker) le Soi empirique des finitistes, mais le Soi idéal, non psychologique, le concept de Soi. Citons l'extrait, très célèbre, de la lettre à Weber (Ewald, 1996, p. 835) : $^{40}$ Nous sommes une race divine

40. Nous aurions pu également convoquer l'extrait du Nachlass publié dans Ewald, 1996, p. 837: «De tous les moyens que l'esprit humain a créés jusqu'à présent pour simplifier sa vie c'est-à-dire pour simplifier le travail en quoi consiste le penser - aucun n'est d'aussi grande importance et n'est autant rattaché à la nature la plus intime de l'esprit que le concept de nombre. L'arithmétique, dont le seul objet est ce concept, est déjà aujourd'hui une science dont l'ampleur est immense, et il ne peut y avoir aucun doute qu'il n'y a absolument aucune limite à son développement futur; et le domaine de ses applications est également immense, car chaque homme, même s'il ne le saisit pas clairement, est un homme des nombres, un arithméticien.» 
et nous possédons, sans aucun doute, un pouvoir créateur, non pas simplement dans les choses matérielles (chemin de fer, télégraphe), mais surtout dans les choses spirituelles. » La référence au divin éloigne ici toute interprétation empirique, psychologique, du Soi.

Résumons. Même si aucun élément ne permet d'affirmer l'existence d'une influence directe de la doctrine idéaliste du Soi, la lecture que Royce propose de Dedekind, 1888, ne nous semble pas, pour au moins deux raisons, invraisemblable. D'une part, certains indices, qu'il conviendrait certes d'étudier de plus près, semblent attester une reprise par le mathématicien de concepts et de thèmes présents dans les écrits du philosophe H. Lotze, amorçant alors un retour vers l'idéalisme. D'autre part, le lien tissé entre nombres et Soi idéal permet de tenir ensemble les principales pièces du puzzle que constitue Die Zablen: le «logicisme » de Dedekind et le refus de fonder l'arithmétique sur une intuition pure du temps, son insistance sur la création et l'activité humaine, la place centrale de l'arithmétique dans les mathématiques, le rôle du $\mathbb{S} 66$. Il reste que cette interprétation purement "métaphysique » force, répétons-le, la lettre de Die Zablen, en faisant jouer un rôle central à la preuve de l'existence d'un ensemble infini; le monde de mes pensées n'est plus conçu comme seulement un exemple de système infini, mais comme la matrice de tous les systèmes simplement infinis.

\section{Conclusion : mathématiques et métaphysique chez Bradley, Royce et Russell}

Revenons, pour conclure, à Bradley et aux lectures critiques que Russell et Royce en ont fait. Le point commun entre Russell et Royce est qu'ils se sont servis des nouvelles mathématiques pour critiquer Appearance and Reality. Mais, si Russell prétend que seule une métaphysique réaliste, débarrassée de tout préjugé psychologique, est susceptible de rendre compte des nouvelles doctrines, Royce, lui, voit dans le raisonnement de Dedekind une occasion de renouveler l'idéalisme. Peut-on imaginer deux approches plus opposées que celles-ci? Ce choc frontal pourrait, dans un premier temps du moins, redonner des couleurs à la philosophie de Bradley : le fait que les mêmes travaux mathématiques engendrent des interprétations aussi divergentes ne montre-t-il pas que la rationalité métaphysique n'a rien à voir avec la légalité mathématique? Et, dans ces conditions, la position, prudente, de Bradley, qui consiste à la fois à confesser son "inaptitude» pour l' "abstraction » mathématique, et à tenir bon sur la recherche des premiers principes, c.-à-d. la métaphysique, n'est-elle pas finalement la plus raisonnable?

La lecture de la correspondance Bradley-Russell est à cet égard édifiante. L'auteur de Appearance and Reality, tout en reconnaissant la compétence de Russell dans le domaine logique et mathématiques, ne cesse de lui reprocher son manque de cohérence — Bradley frappe d'ailleurs souvent juste ${ }^{41}$ : 
qu'en est-il, dans les Principles, de l'unité de la proposition? Quel est le statut exact de la notion de classe? Peut-on réellement soutenir que la différence est une relation? Bradley, ayant confessé son inaptitude pour les mathématiques, semble, pour ainsi dire, demander à Russell qu'il fasse de même dans le domaine de la métaphysique ${ }^{42}$. L'idée qu'il y a une rationalité purement métaphysique, différente des autres formes de rationalité, gouverne les attaques que le philosophe idéaliste adresse à celui qui fut l'un de ses disciples.

Cette attitude bradleyenne, consistant à distinguer différents domaines de rationalité qui ne se recouvrent pas, nous semble excessivement timorée. Notons d'abord que l'ignorance n'oblige jamais : l'inaptitude aux mathématiques ne saurait être le gage d'une quelconque compétence en métaphysique $^{43}$. Mais plus profondément, l'idée que métaphysique et mathématiques seraient des domaines juxtaposables, sans lien les uns avec les autres, et que donc la politesse de ne pas empiéter sur le territoire des voisins devrait être considérée comme une vertu intellectuelle, est profondément étrange et inutilement pusillanime. C'est certainement, entre autres, à Bradley que Russell pense lorsqu'il écrit le dernier chapitre de Problems of Philosophy ${ }^{44}$, dans lequel il dénonce les pensées timorées, égocentrées. Chez Bradley, ce n'est certes pas «le désir de prouver la parenté du monde et du Moi » qui est à l'origine d'une auto-affirmation stérile, puisque le philosophe anglais veut montrer précisément le contraire, l'altérité de la pensée et de la réalité. Mais le scepticisme de Bradley ressortit de la même étiologie: la volonté de satisfaire "son" intellect et "ses" demandes, sans chercher à l'ajuster et à l'aiguiser au contact d'une positivité extérieure, ici les mathématiques.

En revanche, Russell se trompe lorsqu'il englobe dans sa même critique l'ensemble de l'idéalisme. La belle analyse de Royce manifeste que le refus de se confronter aux mathématiques n'est absolument pas l'apanage

42. Cela ressort de la façon dont Bradley présente sa critique de Russell dans Bradley, 1914, p. 280 : « Je vais maintenant faire quelques remarques au sujet de certaines des idées fondamentales utilisées par M. Russell, et je vais tenter de montrer que ces idées contiennent des contradictions (inconstencies). Je ne suis, en un sens, pas à la hauteur de cette tâche. Je suis complètement incompétent pour évaluer la grande œuvre de M. Russell (Principles of Mathematics). Mais, si la partie mathématique est aussi bonne que la partie philosophique, je suis certain qu'il a produit un livre d'une valeur singulière. Me restreindre à une critique unilatérale d'idées que je ne peux que partiellement comprendre est ingrat de ma part, et je ne le ferais pas si je ne me sentais pas en un sens obligé de le faire." Derrière la rhétorique, on retrouve l'idée d'un partage possible, entre d'une part, ce qui relève des mathématiques, et de l'autre, ce qui relève de la seule métaphysique.

43. Bradley fait très souvent appel à l'introspection et à l'expérience "concrète » dans ses critiques de Russell. Des formules comme «I cannot follow any train of reasoning which is highly abstract [...] " reviennent très souvent. Ce trait est profondément lié à l'idée que Bradley se fait de la métaphysique, comme saisie d'une totalité qui n'est donnée que dans l'immédiateté. Le philosophe semble ainsi penser que son inaptitude aux mathématiques, qui sont par essence «abstraites ", est la conséquence de son sens du concret et de la totalité, c'est-à-dire de son talent métaphysique.

44. Voir Russell, 1912, p. 177-185. 
de l'idéalisme et que la générosité n'est donc pas non plus le propre de l'atomisme logique. La proximité est en effet, au final, plus grande entre Royce et Russell qu'elle ne l'est entre les deux idéalistes. Si Royce et Russell s'opposent sur la question de savoir quelle métaphysique fournit le cadre naturel des nouvelles mathématiques, ils s'accordent tous les deux en effet, contre Bradley, pour reconnaître que l'articulation entre les deux sphères est une exigence rationnelle $e^{45}$. La métaphysique, qu'elle soit idéaliste ou qu'elle soit réaliste, ne peut, selon Royce et Russell, ignorer la positivité mathématique. Quant à savoir laquelle des deux interprétations parvient à «replacer la recherche de Dedekind dans sa relation la plus naturelle à l'enquête métaphysique générale ", la question reste ouverte - mais l'essentiel n'est-il pas ailleurs: dans la reconnaissance partagée d'une tâche à accomplir ?

\section{Références}

Allard, James, The Logical Foundations of Bradley's Metaphysics. Judgment, Inference, and Truth, Cambridge, Cambridge University Press, 2005.

Bolzano, Bernard, Les paradoxes de l'infini, Paris, Seuil, 1993.

Borges, José-Luis, Enquêtes (1937-1952), Paris, Gallimard, 1957.

Bradley, Francis Herbert, Appearance and Reality, Oxford, Clarendon Press, 1893.

- Essays on Truth and Reality, Oxford, Clarendon Press, 1914.

Cantor, Georg, "Beiträge zur begründung der transfiniten mengenlehre ", Mathematische Annalen, 46, 1895, p. 481-512; 1897, 49, p. 207-246.

Dedekind, Richard, Was sind und was sollen die Zablen?, Braunschweig, Vieweg, 1888.

Dugac, Pierre, Richard Dedekind et les fondements des mathématiques, Paris, Vrin, 1976.

Ewald, William, dir., From Kant to Hilbert: A source book in the foundations of mathematics, Oxford, Oxford University Press, 1996.

Ferreiròs, José, Labyrinth of thought. A history of set theory and its role in modern mathematics, Berlin, Birkhäuser Verlag, 1999.

Fichte, Johann Gottlieb, Euvres choisies de Philosophie première (1794-1797), Paris, Vrin, 1964.

Grattan-Guinness, Ivor, The Search for Mathematical Roots 1870-1940. Logics, set theories and the foundations of mathematics from Cantor through Russell to Gödel, Princeton, Princeton University Press, 2000.

Griffin, Nicholas, Russell's Idealist Apprenticeship, Oxford,Clarendon, 1991.

Hegel, Georg Wilhelm Friedrich, Encyclopédie des sciences philosophiques, volume III, Philosophie de l'Esprit, Paris, Vrin, 1988.

Hilbert, David, «Neubegründung der mathematik », Abhandlungen mathematischen Seminar Universität Hamburg, 1, 1922, p. 157-177.

Kant, I. Anthropologie du point de vue pragmatique, Paris, Vrin, 1988.

45. Dans la lettre de Russell à Couturat du 4 juillet 1905 (Schmid, 2001, p. 509), on relève ce jugement : «Royce est assez habile pour un philosophe, mais il pense qu'on peut faire des systèmes dans le gros, sans examiner minutieusement chaque partie, et c'est ce que mon expérience ne me permet pas de croire.» 
Leibniz, Gottfried Wilhelm, Recherches générales sur l'analyse des notions et des vérités. 24 thèses métaphysiques et autres textes logiques et métaphysiques, Paris, Presses Universitaires de France, 1998.

Lotze, Hermann, Metaphysique, trad. française de A. Duval, Paris, Firmin-Didot, 1883.

—. Geschichte der deutchen Philosophie seit Kant - Diktate aus den Vorlesungen, Hirzel, 1882.

McTaggart, John McTaggart Ellis, Philosophical Studies, New York, Freeport, 1968. Royce, Josiah, The World and the Individual, Goucester Mass., Peter Smith, 1901. Russell, Bertrand, The Principles of Mathematics, London, Routledge; 1903.

—. The Problems of Philosophy, Oxford, Oxford University Press, 1912. Introduction to Mathematical Philosophy, Londres, George and Allen, 1921.

Schmid, Anne-Françoise, dir., Bertrand Russell / Louis Couturat (1897-1913): Correspondance sur la philosophie, la logique et la politique, Paris, Kimé, 2001.

Van Heijenoort, Jean, dir., From Frege to Gödel. A source book in mathematical logic, 1879-1931, Harvard, Harvard University Press, 1967. 